\title{
DAS BANNWABENVERFAHREN ALS ELEMENT IMKERLICHER BETRIEBSWEISE BEI STARKEM BEFALL MIT VARROA JACOBSONI OUD.
}

\author{
V. MAUL, A. KLEPSCH, U. ASSMANN-WERTHMÜLLER \\ Hessische Landesanstalt für Tierzucht, Abteilung für Bienenzucht D-3575 Kirchhain 1
}

\section{ZUSAMMENFASSUNG}

Der Einfluß des Bannwabenverfahrens auf die Entwicklung und Leistung von schwach befallenen Carnicavölkern wurde in 2 Versuchen überprüft. In einem dieser Versuche wurde auch der Zeitbedarf für die Durchführung des Verfahrens ermittelt. Darüber hinaus wurde ein Langzeitversuch eingeleitet mit dem Ziel, die Möglichkeiten der Verringerung des notwendigen Einsatzes chemischer Mittel zu prüfen, wenn das Bannwabenverfahren als Element der Betriebsweise bei starkem Varroabefall regelmäßig angewendet wird.

Unter mitteleuropäischen Verhältnissen mit meist im Juli endender Sommertracht beeinflußt die Bannwabenbehandlung die Leistungsfähigkeit der Völker nicht nachteilig, sofern die Behandlung jeweils am Höhepunkt der Volksentwicklung eingeleitet wird. Der Zeitbedarf für die ersten beiden Behandlungsschritte ist etwa doppelt so hoch wie für normale Inspektionen. Die feste Einordnung dieses Verfahrens in die Betriebsweise ermöglicht es, den Bedarf für chemische Behandlungen bei starkem Varroabefall crheblich zu reduzieren, kann diesen jedoch nich vollständig ersetzen.

\section{EINLEITUNG}

Seit dem Übergreifen des Parasiten Varroa jacobsoni Oud. auf Apis mellifera $\mathrm{L}$. kann die wirtschaftliche Bienenhaltung in den betroffenen Ländern nur durch Einsatz chemischer Bekämpfungsmittel zur regelmäßigen Befallsdämpfung aufrechterhalten werden. Eine sichere Befallstilgung ist mit den bisher verfügbaren Mitteln ebensowenig möglich wie eine Verhinderung der weiteren Ausbreitung. Der Kosten- und Arbeitsaufwand für die Bekämpfungsmaßnahmen wird in der Imkereiwirtschaft inzwischen weitgehend als unvermeidlich hingenommen. Auf lange Sicht birgt diese Strategie der Bekämpfung mit chemischen Mitteln jedoch grundsätzlich zwei Risiken :

1. Die Möglichkeit einer Resistenzentwicklung des Parasiten, die je nach Wirkstoffart und Anwendungsweise unterschiedlich einzuschätzen ist. 
2. Die Möglichkeit einer allmählich steigenden Belastung der wichtigsten Bienenprodukte Honig und Wachs durch Rückstände von Behandlungsmitteln, die wiederum je nach Wirkstoffart und Anwendungsmodus unterschiedlich einzuschätzen ist.

Es erscheint deshalb als dringend notwendig, nach alternativen Strategien zu suchen, welche geeignet sind, die Abhängigkeit von chemischen Bekämpfungsmethoden zu verringern. Einer züchterischen Veränderung der Biene im Sinne einer gesteigerten Widerstandskraft konnten angesichts der massiven flächenhaften Schadensverläufe auch auf längere Sicht kaum Erfolgschancen eingeräumt werden. Erfolgreicher waren dagegen verschiedene Versuche, durch bestimmte betriebstechnische Eingriffe - sogenannte biotechnische Maßnahmen - die Parasitenvermehrung im Volk zumindest befristet einzuschränken. So wird z.B. die Trennung der schwächer befallenen Bienen von der stärker befallenen Brut im sogenannten Kunstschwarmverfahren sehr häufig als unterstützende Maßnahme praktiziert (PFEFfERLE, 1983). Bei noch relativ geringem Befallsgrad wirkt das regelmäßige Entfernen verdeckelter Drohnenbrut nachhaltig (ScHulz et al., 1983). Als wirksamste dieser biotechnischen Maßnahmen hat sich bisher das sogenannte Bannwabenverfahren erwiesen. Dabei wird nacheinander 3-4 mal mittels Absperrgittertasche die Legetätigkeit der Königin auf je 1 Wabe beschränkt. Diese Waben werden nach der Verdeckelung und vor dem Schlüpfen der Bienen entfernt und vernichtet.

Die Methode war ursprünglich konzipiert, um Bienenvölker für Versuchsbehandlungen brutfrei zu machen (RUTTNER et al., 1980). In mehreren Untersuchungen konnte jedoch die auffällig starke milbenentziehende Wirkung dieser Verfahrensweise alleine belegt werden (Ruttner und Koeniger, 1979 ; Maul, 1983a ; Klepsch und Maul, 1983). Sie erschien deshalb als besonders geeignet, bei starkem Parasitenbefall eine Schädigung der Völker während der Trachtzeit auszuschließen, ohne das Risiko einer Rückstandsbelastung der Honigernte einzugehen. Einer breiteren Anwendung in der Praxis stellten sich jedoch als Hindernisse die negative Einschätzung des erforderlichen Arbeitsund Zeitaufwandes und die Unsicherheit über mögliche negative Auswirkungen auf die Entwicklungs- und Leistungsfähigkeit der Bienenvölker entgegen. Der vorliegende Beitrag berichtet über erste Untersuchungen zum praktischen Einsatz des Bannwabenverfahrens. 


\section{MATERIAL UND METHODEN}

a) Technik des Bannwabenverfahrens im 9-Tage-Rhythmus

Zum befristeten Absperren werden einfach herzustellende Rähmchen mit Absperrgitter verwendet (Abb. 1). Sie decken die Wabenflächen zwischen den Seitenträgern (Hoffmann-Rähmchen) ab und werden durch leicht lösbare Drahtschleifen oder U-Klammern zusammengehalten. Die Leerwabe muß mindestens einen offenen Durchgang von einer Seite zur anderen haben.

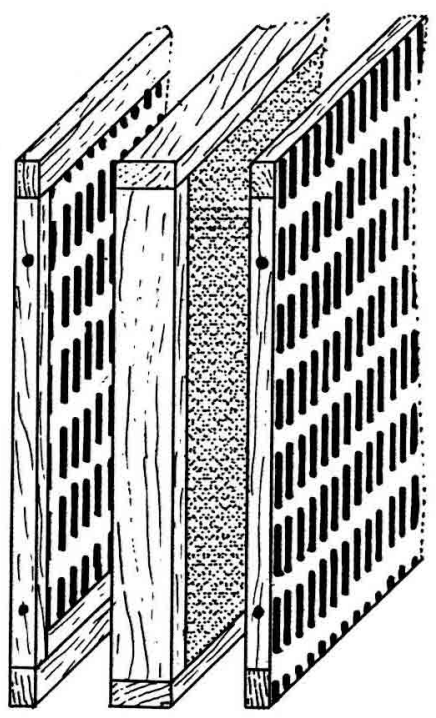

a

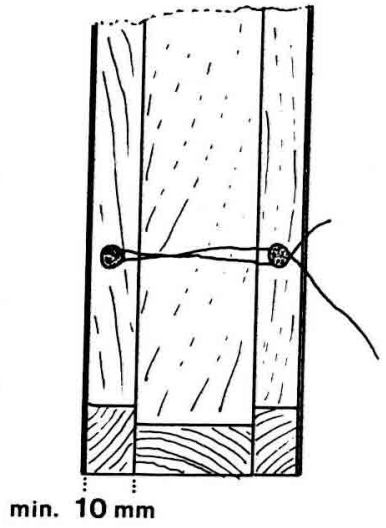

b



C

AвB. 1 a-c. - Absperrgitterrahmen für das Bannwabenverfahren.

a) Wabe mit zwei Gitterrahmen; b) einfache Befestigung der Gitterrahmen mittels Drahtschlingen; c) Ansicht von oben : Einfügen der Gitterrahmen zwischen die Seitenträger bei « Hoffmann "-Rähmchen.

FIG. 1 a-c. - Excluder frames for the trapping-comb-technique.

a) Comb with two excluder frames ; b) simple fastening of the excluder frames by threads of comb wire ; c) view from top : insertion of the excluder frames between the end bars of "Hoffmann » frames.

\section{Arbeitsgang (Tag 0):}

Königin in die einseitig offene Wabentasche I setzen (zweckmäßig durch eine Wabenlücke auf die bereits abgedeckte Gegenseite laufen lassen) und Tasche schließen. Volk gründlich auf Weiselzellen durchsehen. Zwei nicht benötigte Waben entfernen, um Platz für die Tasche zu gewinnen und Tasche im oberen Bereich des Brutnestes einstellen. 


\section{Arbeitsgang (Tag 9):}

Königin wic oben in die Wabentasche auf Leerwabe II übertragen. Volk nochmals gründlich auf Weiselzellen kontrollieren. Eine weitere Wabe entfernen. Wabentasehe wieder einstellen, daneben die nun bebrütete Bannwabe I zur Verdeckelung offen cinstellen.

\section{Arbeitsgang (Tag 18) :}

Königin in Wabentasche auf Lecrwabe III übertragen. Bannwabe II zur Verdeckelung frci cinstellen. Bannwabe I (verdeckelt, kurz vor Schlupf) zur Vernichtung entnehmen. Eine Kontrolle auf Weiselzellen ist im freien Brutnest nicht mehr erforderlich, jedoch evtl. auf den Bannwaben.

\section{Arbeitsgang ( $T a g$ 27) :}

Königin wieder zur freien Eiablage entlassen bzw. durch Jungkönigin ersetzen. Bannwabe II zur Vernichtung entnehmen. Bannwabe III zur Verdeckelung frei cinstellen und Lecrwabe oder Mittelwand zugeben. Alle vorher frei angelegte Brut ist zu dieser Zcit bereits ausgelaufen, so daß bedarfsweise überalterte Waben des Brutnestes gegen jüngere ausgetauscht werden können.

\section{Arbeitsgang ( $\operatorname{Tag} 36)$ :}

Kontrolle auf Weiselrichtigkeit, Entnahme von Bannwabe III zur Vernichtung. Zugabe einer Leerwabe oder Mittelwand.

Infolge der ea. 3-wöchigen Unterbrechung des Jungbienennachschubs sind in der folgenden Zeit Maßnahmen zur Schwarmkontrolle nicht mehr crforderlich (ausführliche Beschreibung s. MaUL, 1983b).

\section{b) Prüfung des Einflusses auf die Volksentwicklung und den Honigertrag}

Auf mehreren benachbarten Ständen mit vergleichbarer Höhen- und Trachtlage wurde jeweils ein Teil der Völker dem Bannwabenverfahren unterzogen. Die übrigen Völker wurden als Kontrolle in der bei Zuchtwertprüfungen üblichen Betriebsweise geführt: Brutnest auf 2 Zargen á 10 Zanderwaben : Aufsetzen von Honigzargen nach Bedarf; während der Schwarmzeit Inspektion im Rhythmus von 9 Tagen. Es wurden ausschließlich Carnica-Reinzuchtvölker mit künstlich besamten Königinnen verschicdener Linien bzw. Linienkombinationen benutzt. Der Varroabefall war auf diesen Ständen noch sehr gering. Dic Versuchsgruppe wurde im Hinblick auf mögliche technisch bedingte Ausfälle stärker besetzt. Die verfügbaren Völker wurden nach Herkunft und Entwicklungsstand so verteilt, daß beide Gruppen annähernd symmetrisch aufgebaut waren.

Folgende Beurteilungskriterien wurden registriert : Die Volksstärke zu Bchandlungsbeginn, vor der Einwinterung im Behandlungsjahr und zum Trachtbeginn im Folgejahr, der Honigertrag im Behandlungsjahr und im Folgcjahr.

\section{c) Bestimmung des Zeitaufwandes}

Im Verlauf eines Prüfjahres wurden genaue Zeitmessungen bei der Durchführung der Arbeitsgänge zwischen Behandlungsbeginn und Behandlungsende an Versuchs- und Kontrollvölkern durchgeführt.

\section{d) Langzeitversuch mit Bannwabenverfahren als einziger Maßnahme der Varroakontrolle}

Auf einem im stark befallenen Gebiet gelegenen Versuchsstand wurden alle vorhandenen Bienenvölker jeweils gleichzeitig dem Bannwabenverfahren nach dem $3 \times 9$-Tage-Rhythmus unterzogen. Verdeckkelte Drohnenbrut wurde nicht systematisch entfernt. Die sonst obligatorische Behandlung im Herbst mit chemischen Mitteln unterblieb. Zur Überwachung der Befallsentwicklung wurde der natürliche Milbentotenfall mittels gazcgesicherter Bodencinlagen ab Mai 1985 ganzjährig und vollständig erfaßt (Intervalle nach Jahreszeit und Arbeitsmöglichkeit wechselnd). Im Sommer 1985 wurden 5 Völker nach Ende der Behandlung zur Bestimmung der Wirksamkeit abgetötet und im Frühjahr 1986 durch neu eingestelte Völker ersetzt. Der Versuch wurde mit Carnica-Völkern nicht definierter Herkunft begonnen. Im Juli 1986 wurden natürlich gepaarte Königinnen aus 3 definierten Carnica-Herkünften eingeweiselt. Dic Magazinbeuten (Zarge á 10 Zanderwaben) waren in enger Reihe in einem Bienenhaus aufgestellt. weshalb Verdriftung zwischen Nachbarvölkern nicht ausgeschlossen werden kann. 


\section{ERGEBNISSE}

\section{Prüfung des Einflusses auf die Volksentwicklung und den Honigertrag}

Die Ergebnisse zweier Versuchsreihen sind in den Tabellen 1 und 2 zusammengefaßt. Zu Beginn der ersten Versuchsserie (1985) war der Entwicklungsstand der einzelnen Völker nach schwieriger Überwinterung noch sehr unterschiedlich. Der Behandlungsbeginn war durch aufkommende Schwarmneigung bei den stärksten Völkern bestimmt. Im Interesse der Gleichzeitigkeit der Behandlung wurden auch noch schwächer entwickelte Völker einbezogen. Das Frühtrachtangebot (Raps) war mäßig. Die eigentliche Sommertracht, deren Ernte durch die Behandlung hätte beeinflußt werden können, fiel infolge anhaltend regnerischer Witterung nahezu völlig aus. Unter diesen ungünstigen Witterrungsbedingungen war die nachfolgende Einwinterungsstärke bei der Versuchsgruppe im Durchschnitt geringer als bei der Kontrollgruppe. Der Unterschied ist jedoch statistisch nicht abzusichern. Ein Unterschied im gleichen Sinne zeigt sich auch beim Entwicklungsstand zum Trachtbeginn des Folgejahres und der Ertragsleistung unter sehr günstigen Trachtverhältnissen. Diese Unterschiede lassen sich aber statistisch ebenfalls nicht absichern. 5 Versuchsvölker fielen wegen Königinnenverlusts im Spätsommer bzw. Winter aus, ein Volk ging im Winter ein.

ТАВ. 1. - Stärke und Honigertrag von Versuchs- und Kontrollvölkern (Versuch 1985)

Bannwabe $3 \times 9$ Tage : 10.06.-08.07.1985

TABL. 1. - Strength and honey yield of experimental and control colonies (experiment 1985) Trapping comb $3 \times 9$ days : 10.06-08.07.1985

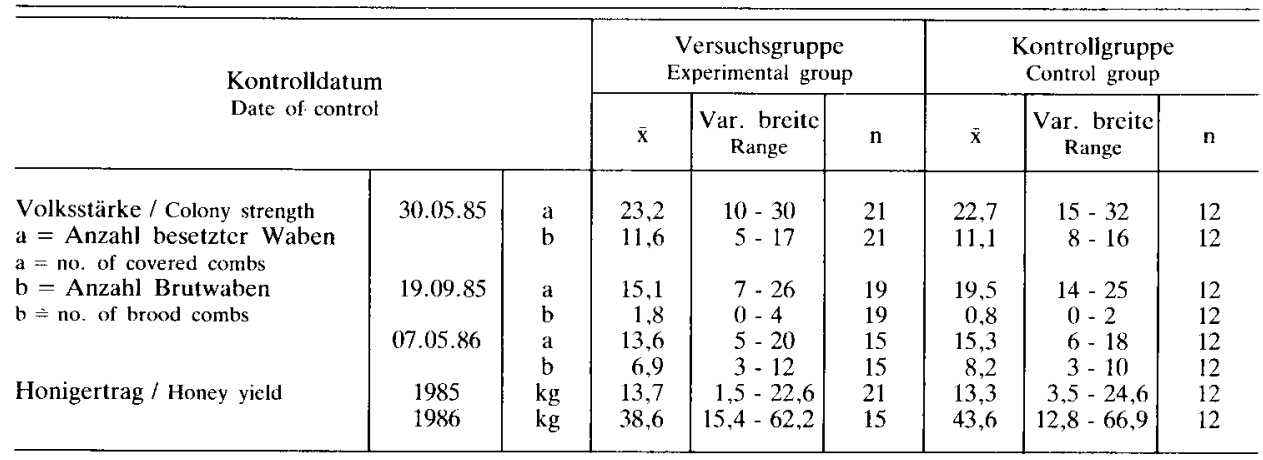

Die Unterschiede sind nicht signifikant / The differences are not significant.

Anmerkung zu Völkerverlusten : 2 Völker nach mißglückter Umweiselung im Sommer 85 aufgelöst. 3 Völker im Winter $85 / 86$ weisellos, 1 Volk im Winter tot.

Note to colony losses : 2 colonies removed after failure of requecning. 3 colonies queenless in winter 85/86. 1 colony dead in winter. 
Die zweite Versuchsserie konnte 1986 unter wesentlich günstigeren Witterungs- und Trachtverhältnissen eingeleitet werden. Die Völker beider Gruppen waren bei Behandlungsbeginn besser und einheitlicher entwickelt. Der Honigertrag war bei der Versuchsgruppe im Durchschnitt besser als bei der Kontrollgruppe. In der Volksstärke bei der Einwinterung und zum Trachtbeginn des Folgejahres und in der Leistung des Folgejahres zeigte sich wieder die umgekehrte Tendenz. Die Unterschiede sind jedoch in keinem Falle statistisch abzusichern. Die Trachtsituation war 1987 ähnlich wie 1985 (nur mäßiges Frühtrachtangebot, Sommertracht verregnet). Aus technischen Gründen mußte der Leistungsvergleich für 1987 auf Wägedaten der ganzen Stöcke gestützt werden, die jedoch erfahrungsgemäß gut mit den späteren Schleuderergebnissen korrelieren. Zwei Überwinterungsverluste dieser Versuchsserie waren auf Drohnenbrütigkeit der Königin zurückzuführen.

TAB. 2. - Stärke und Honigertrag von Versuchs- und Kontrollvölkern (Versuch 1986) Bannwabe $3 \times 9$ Tage : 16.06.-14.07.1986

TABL. 2. - Strength and honey yield of experimental and control colonies (expcriment 1986) Trapping comb $3 \times 9$ days : 16.06-14.07.1986

\begin{tabular}{|c|c|c|c|c|c|c|c|c|}
\hline \multirow{2}{*}{\multicolumn{3}{|c|}{$\begin{array}{l}\text { Kontrolldatum } \\
\text { Date of control }\end{array}$}} & \multicolumn{3}{|c|}{$\begin{array}{l}\text { Versuchsgruppe } \\
\text { Experimental group }\end{array}$} & \multicolumn{3}{|c|}{$\begin{array}{l}\text { Kontrollgruppe } \\
\text { Control group }\end{array}$} \\
\hline & & & $\overline{\mathbf{u}}$ & Var. breite & & & Var. breite & \\
\hline $\begin{array}{l}\text { Volksstärke / Colony strength } \\
a=\text { Anzahl besetzter Waben } \\
a=\text { no, of covered combs } \\
b=\text { Anzahl Brutwaben } \\
b=\text { no. of brood combs }\end{array}$ & $\begin{array}{c}16.06 .86 \\
17.09 .86 \\
11.05 .87 \\
1986 \\
23.04- \\
01.06 .1987\end{array}$ & $\begin{array}{l}\mathrm{a} \\
\mathrm{b} \\
\mathrm{a} \\
\mathrm{b} \\
\mathrm{a} \\
\mathrm{b} \\
\mathrm{kg} \\
\mathrm{kg}\end{array}$ & $\begin{array}{r}28,6 \\
12,3 \\
17,7 \\
1,8 \\
15,3 \\
10,1 \\
44,9 \\
14,6\end{array}$ & $\begin{array}{r}21-36 \\
8-18 \\
8-24 \\
0-4 \\
8-18 \\
5-13 \\
12,8-66,9 \\
4,1-22,0\end{array}$ & $\begin{array}{l}18 \\
18 \\
\\
18 \\
18 \\
18 \\
16 \\
16 \\
18 \\
16\end{array}$ & $\begin{array}{r}28,6 \\
13,3 \\
19,2 \\
0,6 \\
18,3 \\
11,3 \\
36,9 \\
15,5\end{array}$ & $\begin{array}{c}20-35 \\
9-17 \\
14-26 \\
0-3 \\
16-23 \\
9-15 \\
16,3-58,9 \\
7,6-19,8\end{array}$ & $\begin{array}{l}10 \\
10 \\
10 \\
10 \\
10 \\
10\end{array}$ \\
\hline
\end{tabular}

Die Unterschiede sind nicht signifikant / The differences are not significant.

Anmerkung zu Völkerverlusten : 2 Völker wurden wegen Drohnenbrütigkeit der Königin aufgelöst.

Note to colony losses : 2 colonies were removed because of drone laying queens.

\section{Bestimmung des Zeitaufwandes}

Die Ergebnisse von insgesamt 6 Zeitmessungen aus dem Jahr 1986 sind in Abb. 2 graphisch dargestellt. Helle Säulen bezeichnen die Kontrollgruppe, schraffierte Säulen die Versuchsgruppe. Zu den Mittelwerten wird die Standardabweichung graphisch dargestellt. 


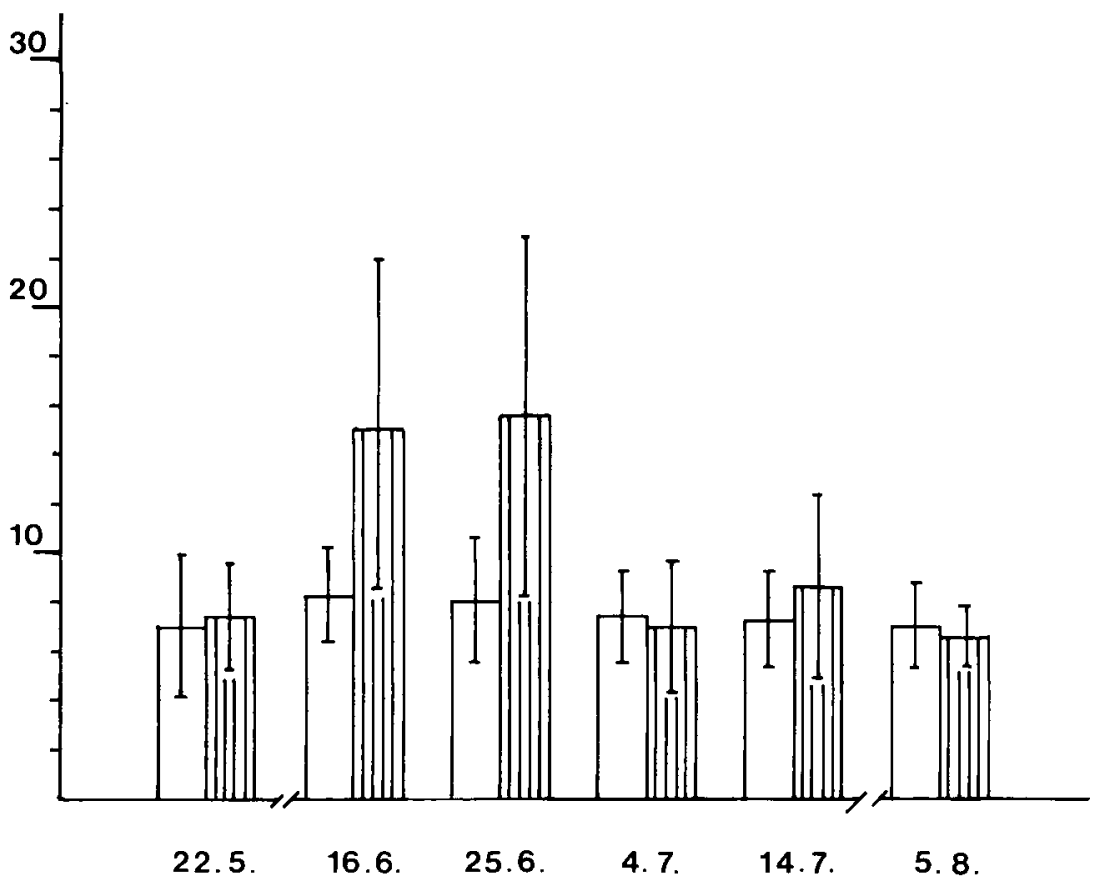

Авв. 2. - Vergleich der Arbeitszeit für das Bannwabenverfahren und normale Arbeitsgänge.

Abszisse: Arbcitstermine 1986.

Ordinate: Durchschnittliche Arbeitszeit je Volk in Minuten ; dunkle Säulen = Versuchsgruppe, helle Säulen $=$ Kontrollgruppe, senkrechte Linien $=$ Standardabweichung $(\mathrm{s})$.

Kurzbeschreibung der Arbeitsgänge: 22.5. und 5.8. = normale Inspcktionen in beiden Gruppen (zu Vergleichszwecken), cbenso alle Arbeitsgänge in der Kontrollgruppe. Versuchsgruppe : 16.6. = Suchen und Sperren der Königin auf Wabe I, Kontrolle auf Schwarmzellen ; 25.6. = Wechsel der Königin auf Wabe II, Kontrolle auf Schwarmzellen; 4.7. = Wechsel der Königin auf Wabe III, Entnahme von Wabe I; 14.7. = Freisetzen der Königin bzw. Umweiseln, Entnahme von Wabe II. (Für dic Entnahme von Wabe III wurde die Zeit nicht crfaßst).

FIG. 2. - Comparison of working time for the trapping-comb-technique and regular inspections.

Abscissa: Working datcs 1986.

Ordinate: Average working time per colony in minutes ; dark columns = experimental group, light columns $=$ control group, vertical bars $=$ standard deviation $(\mathrm{s})$.

Short description of procedures : 22.5. and 5.8. = regular inspections in both groups (for comparison) as well as all dates for the control group. Experimental group : 16.6. = searching and arresting of the queen to comb $\mathrm{I}$, control on swarm cells ; 25.6. = change of queen to comb II, control on swarm cells ; 4.7. = change of queen to comb III, removing of comb $\mathrm{I} ; 14.7$. = queen set free again (eventually replaced by a young one), removing of comb 11. (The time for the removal of comb III was not registered).

Bei dem ersten und letzten Termin handelt es sich um Vergleichswerte von Inspektionen, die für alle Völker gleichartig verliefen. Sie lassen erwartungsgemäß keine Unterschiede erkennen. Der Zeitaufwand für die Versuchsvölker ist dagegen beim ersten und zweiten Schritt der Behandlung (16.06. und 

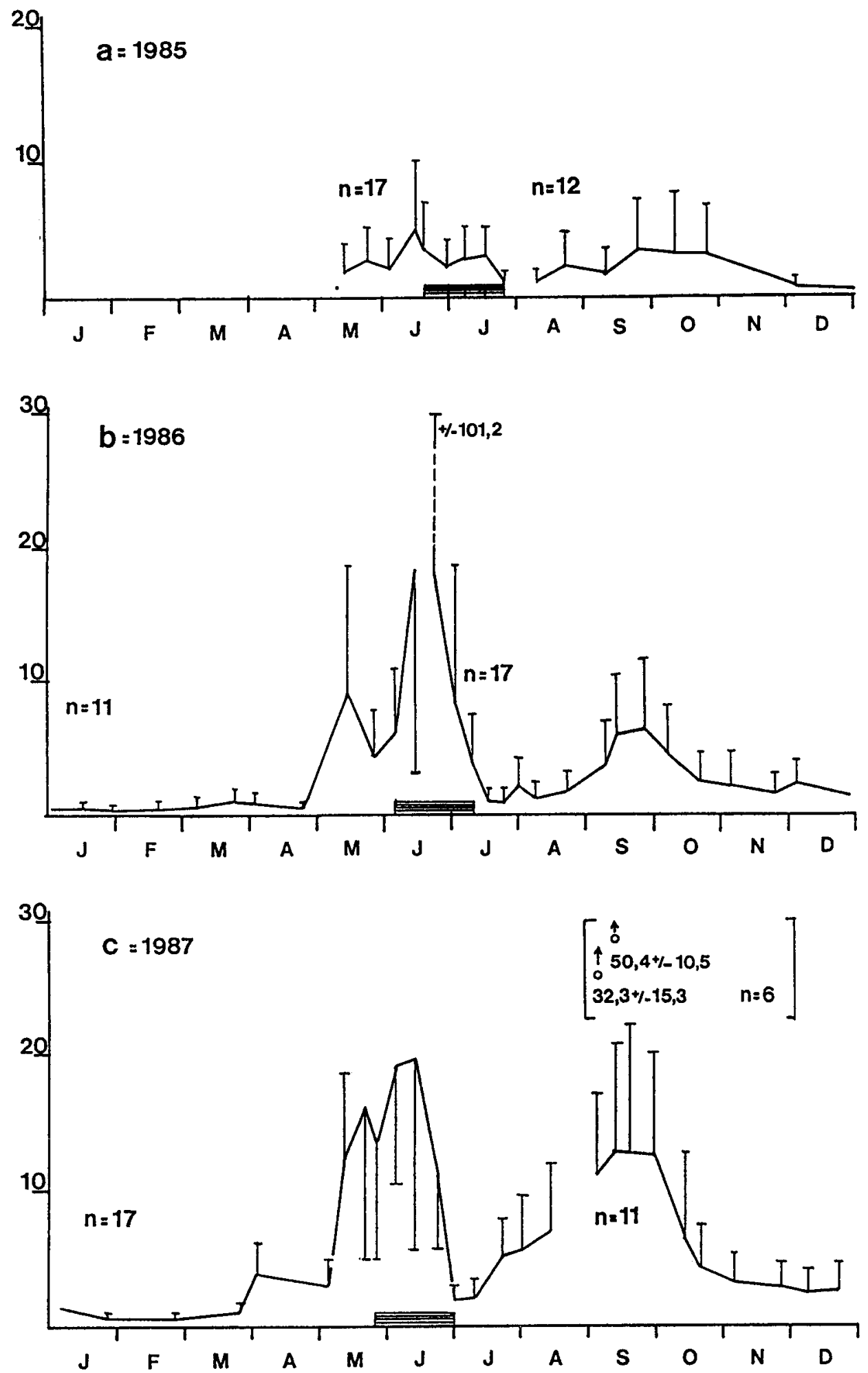
25.06.) im Durchschnitt nahezu doppelt so hoch wie bei den Kontrollvölkern. Dies erklärt sich aus der Notwendigkeit, die zunächst frei im Volk laufende Königin erstmals auszufangen und in die Gittertasche $\mathrm{zu}$ sperren, sowie an beiden Terminen das Volk sehr gründlich auf Weiselzellen zu untersuchen. Der hier ermittelte absolute Zeitbedarf für " normale » Arbeitsgänge darf nicht als repräsentativ für eine reine Wirtschaftsimkerei gelten. Er ist deshalb höher, weil im Rahmen der Versuchsdurchführung stets sehr genaue Inspektionen für eine jeweils vollständige Datenerhebung erforderlich waren. Der betriebstechnische Vorteil sicherer Schwarmverhinderung wirkte sich 1986 nicht deutlich aus, da die Schwarmstimmung bei allen Völkern bereits durch die sehr gute Tracht unterdrückt wurde.

\section{Langzeitversuch mit Bannwabenverfahren als einziger Maßnahme der Var- roakontrolle}

Als Maßstab für die Entwicklung der Milbenpopulation wird der Verlauf des durchschnittlichen natürlichen Milbentotenfalls je Volk und Tag für die drei Versuchsjahre 1985 bis 1987 in Abb. 3 a-c graphisch dargestellt. 1986 und 1987 hatte sich eine Befallsstärke entwickelt, die ohne den Kontrolleingriff mit hoher Wahrscheinlichkeit zum Eintritt des typischen Schadensverlaufs geführt hätte. Die Zeiten der Bannwabenanwendung sind als schraffierte Balken markiert.

Der natürliche Milbentotenfall zeigt in beiden Jahren einen grundsätzlich ähnlichen Verlauf mit Vorgipfeln im Frühjahr (vom jährlichen Brutbeginn abhängig), sehr starkem sommerlichem Anstieg, sehr starkem Rückgang nach der Bannwabenbehandlung und erneutem Anstieg zum typischen Herbstgipfel. Im sog. Herbstgipfel fallen die Auswirkungen der erneuten Milbenvermehrung und der inneren Umstellung der Brutpflege zusammen. Während der Wintermonate ist der natürliche Milbentotenfall erwartungsgemäß gering. Der Zuwachs setzt 1987 gegenüber 1986 in der sommerlichen und herbstlichen Phase früher ein und ist stärker. Dies läßt auf eine Zunahme der Befallsstärke

\footnotetext{
Aвв. 3 a-c. - Natürlicher Milbentotenfall $1985-1987$ in Völkern mit Bannwabenbehandlung als einziger Maßnahme der Varroakontrolle (keine chemischen Behandlungen).
}

Abszisse: Monat (liegender Balken = Bchandlungszeitraum).

Ordinate: Durchschnittlicher natürlicher Milbentotenfall (n Milben pro Volk und Tag); die Standardabweichung $(s)$ ist nur einseitig aufgctragen.

Fig. 3 a-c. - Natural death rate of mites 1985-1987 in colonies with trapping-comb-technique as the only measure of Varroa control (no chemical treatments).

Abscissa: month (horizontal bar = period of treatment).

Ordinate : average natural death rate of mites ( $\mathrm{n}$ mites per colony and day) ; the standard deviation (s) is shown in one direction only. 
insgesamt schließen. 6 Völker mit extrem starkem Anstieg des Milbentotenfalls im Spätsommer wurden Anfang September 1987 aus dem Bestand genommen und zur Bestimmung der Milbenzahl abgetötet. Sie enthielten im Durchschnitt 6178 Milben. Schadenssymptome waren zu diesem Zeitpunkt noch nicht feststellbar. Die übrigen 11 Völker wurden erneut ohne Herbstbehandlung eingewintert.

Der durchschnittliche Honigertrag belief sich 1986 unter sehr günstigen Trachtverhältnissen auf $47,7 \mathrm{~kg}, 1987$ bei mäßigerem Trachtangebot auf $29,5 \mathrm{~kg}$.

Um die beträchtliche Variation der Befallsstärke zwischen den Völkern zu verdeutlichen, wurde je Volk der natürliche Milbentotenfall in der Zeit zwischen 26.01. und 03.09.1987 kumuliert. Tabelle 3 gibt für die 3 Gruppen definierter Abstammung (Nr. 1-3) und eine nicht definierte Mischgruppe (Nr. 4) die Mittelwerte und Standardabweichungen an. Zusätzlich wird der Anteil unter- und überdurchschnittlicher Werte je Gruppe angegeben.

TAB. 3. - Natülicher Milbentotenfall (kumuliert 26.1.-3.9.1987) in CarnicaVölkern verschiedener Abstammung unter Bannwabenbehandlung

TAB1. 3. - Natural death rate of mites (accumulated 26.1.-3.9.1987) in Carniolan colonies of different origin under trapping-comb-treatment

\begin{tabular}{|c|c|c|c|c|}
\hline Gruppc / Group & 1 & 2 & 3 & $4^{(1)}$ \\
\hline $\begin{array}{l}\text { Summe Milben } \\
\text { Sum of mites }\end{array}$ & $\begin{array}{c}3 \\
2583 \\
645,5\end{array}$ & $\begin{array}{c}6 \\
1627 \\
436,0\end{array}$ & $\begin{array}{c}4 \\
1241 \\
571,0\end{array}$ & $\begin{array}{c}3 \\
1416 \\
492.5\end{array}$ \\
\hline $\begin{array}{l}\text { Anz. Völker über } \varnothing \\
\text { No. of col. above } \varnothing\end{array}$ & 3 & 2 & 1 & 1 \\
\hline $\begin{array}{l}\text { Anz. Völker unter } \varnothing \\
\text { No. of col. below } \varnothing\end{array}$ & 0 & 4 & 3 & 2 \\
\hline
\end{tabular}

(1) Völker nicht definicrter Abstammung.

(1) Colonics of undefined ancestry.

Die Gruppen 1 und 3 unterscheiden sich signifikant (Scheffé-Test : $0.05>\mathrm{P}>0.01$ ).

Groups 1 and 3 are significantly different (Scheffé test : $0.05>\mathrm{P}>0.01$ )

Die unterschiedlichen Befunde deuten auf eine genetische Abhängigkeit der Befallsentwicklung hin. Die Differenz zwischen den Gruppen 1 und 3 ist signifikant (Scheffé-Test, 0,05 > P >0,01). Weder zur individuellen Befallsstärke im Vorjahr noch zum Umfang der Brutentfaltung und Volksstärke lassen sich im Einzelfall bisher klare Beziehungen erkennen. Überraschend ist die Manifestierung dieser Unterschiede auch im Hinblick auf die enge und gemischte Reihenaufstellung. 


\section{DISKUSSION}

\section{Verfahrensweise und mögliche Nebenwirkungen}

Der Arbeitsrhythmus von $3 \times 9$ Tagen deckt bei geringstmöglichem Aufwand den Zeitbedarf für das vollständige Auslaufen der freiangelegten Brut und ca. 1 Woche Übergangszeit für die zuletzt geschlüpften Milben ab. Der gleiche Effekt kann mit etwas mehr Arbeitsaufwand von einem Wochenendimker im Arbeitsrhythmus von $4 \times 7$ Tagen erreicht werden.

Das Arbeiten mit jeweils einer abgesperrten Wabe ist zwar relativ zeitaufwendig, begrenzt aber den Umfang der Bruterzeugung für die Vernichtung. Dies trägt offensichtlich zur Verlängerung der Lebenserwartung der Arbeitsbienen während der «Schlupfpause » bei. Die auch denkbare Absperrung auf mehrere Waben in abgegrenzten Beutenteilen (MAUL, 1983b) würde voraussichtlich zu mehr Energieverschleiß führen. Konkrete Prüfungsergebnisse dieser Verfahrensweise liegen aber noch nicht vor.

Unter den vorherrschenden mitteleuropäischen Trachtverhältnissen liegt die günstigste Zeit für den Behandlungsbeginn zwischen Ende Mai und Ende Juni. Für die im Juli meist auslaufende Sommertracht stehen bei vollentwickelten Völkern noch genügend Jungbienenreserven zur Verfügung. Die nachfolgende "Depression " in der Volksstärke kann noch rechtzeitig vor dem Winter wieder ausgeglichen werden. Für Standorte mit ausgeprägter Spätsommertracht ist das Verfahren nicht geeignet. Abgesehen von einem möglichen Mangel an Sammelbienen wäre auch infolge der Überalterung mit ungenügender Verarbeitung eingetragenen Nektars zu rechnen.

Der Erfolg hängt wesentlich von der Wahl des Starttermins ab. Im Versuch von 1985 war offensichtlich für einige noch schwache Völker die Behandlung zu früh eingeleitet worden. Sie haben die aufgezwungene Brutbeschränkung schlecht verkraftet. Andererseits ist aus Voruntersuchungen bekannt, daß bei fortgeschrittener Schwarmstimmung die Bannwabenbehandlung zu spät kommt und keinen Erfolg bringt, weil die Königin dann nicht mehr ordnungsgemäß legt. Der Wirtschaftsimker kann u.U. durch vorherigen Ausgleich zwischen Völkern oder Austausch von Völkern zwischen Ständen für weitgehend einheitlichen Entwicklungsstand bei der gleichzeitig durchzuführenden Behandlung sorgen.

Die im ersten Versuch gegenüber der Kontrolle erhöhte Ausfallquote- an Königinnen könnte auf eine besondere Belastung durch das Sperrverfahren hindeuten. Witterungsbedingte Aufzuchtmängel der eingesetzten Jungköniginnen sind aber nicht ganz auszuschließen. Notwendige Umweiselungen sollten stets am Ende der Sperrphase erfolgen, nicht später, weil sonst infolge Überal- 
terung des Volkes die Annahme erschwert wird und zusätzliche Brutpausen mit nachteiligen Folgen enstehen.

Die Brutpflege ist nach der Sperrphase sehr intensiv und sicher auch durch vorher nicht genutzte Pollenvorräte im Stock begünstigt. Die Völker verhalten sich wie Jungvölker und brüten im Durchschnitt im Herbst auch etwas länger. Nachteile für die Überwinterungsfähigkeit waren daraus bisher nicht zu erkennen.

Staemmler $(1986,1987)$ kommt in der praktischen Erprobung des Verfahrens in Schleswig-Holstein zu ähnlichen Ergebnissen. Nach einer 1985 relativ spät eingeleiteten Bannwabenbehandlung erzielten seine Versuchsvölker gegenüber den Kontrollen im Folgejahr 1986 einen höheren Ertrag. 1987 war die Tendenz umgekehrt. Im günstigen Trachtjahr 1986 dürfte sich der direkte leistungssteigernde Effekt der termingerechten und befristeten Brutbeschränkung positiv ausgewirkt haben, den schon PREuss (1920) systematisch auszunutzen versuchte. Wieweit die von Kroos (1986) empfohlene Bildung von Zwischenablegern zur Erzeugung der «Fangwaben» praxisgerechte Vorteile bringen kann, bleibt noch zu erproben.

\section{Zeitaufwand für die Durchführung}

Betrachtet man isoliert nur die für die Behandlung erforderlichen Arbeitsgänge, so ist der Mehrbedarf von nahezu $100 \%$ bei 2 Arbeitsgängen relativ hoch. Die hier vorgelegten Daten sollten, wie schon angedeutet, nicht absolut bewertet werden, weil sie durch die Erfordernisse der Versuchsdurchführung zusätzlich belastet sind. Dem Praktiker bieten sich viele Möglichkeiten, durch rationelle Arbeitsroutine zu erheblich geringerem Zeitbedarf zu kommen. Als belastend für einen Wirtschaftsbetrieb steht daneben aber auch die strikte Terminbindung im Vordergrund. Wer darauf eingestellt ist, große Völkerzahlen extensiv zu bewirtschaften, wird ein solches Arbeitsschema nicht ohne weiteres übernehmen können. Man kann aber zu einer günstigeren Bewertung kommen, wenn der nachhaltige schwarmdämpfende Effekt in der Folge der Behandlung und die mögliche Reduzierung sonstiger Maßnahmen der Varroatosebekämpfung berücksichtigt werden. Eine objektive Bewertung sollte deshalb das ganzjährige Konzept der Betriebsweise umfassen. Dafür reichen die bisher gewonnenen Daten noch nicht aus. Sie sind auch schon deshalb nicht zu generalisieren, weil mit sehr sanftmütigem und relativ schwarmträgem CarnicaReinzuchtmaterial gearbeitet wurde. Starke Aggressivität der Völker kann die Durchführung erheblich erschweren. Bei Haltung von schwarmtriebigerem Material könnten eventuell betriebstechnische Vorteile deutlicher werden. Umfangreichere Prüfungen im Sinne eines Betriebsweisenvergleichs erscheinen deshalb als dringend notwendig. 


\section{Reduktion des Einsatzes chemischer Bekämpfungsmittel}

Das Bannwabenverfahren wurde zunächst als eine zusätzliche Maßnahme der Befallsdämpfung verstanden, die in besonderer Weise geeignet ist, bei sehr starkem Varroabefall schon während der Trachtzeit mögliche Schäden sicher auszuschließen. Der nachhaltige Milbenentzug hat dann Überlegungen eingeleitet, wieweit der Einsatz chemischer Bekämpfungsmittel in der noch obligatorischen Herbstbehandlung reduziert werden kann. Die bisherigen Ergebnisse zeigen, daß unter den gegebenen Verhältnissen ein völliger Verzicht auf Medikamente nicht empfohlen werden kann. Auch unter Berücksichtigung einer Sicherheitsschwelle wäre jedoch eine Herbstbehandlung höchstens in jedem zweiten Jahr notwendig gewesen. Mit Rücksicht auf mögliche Standorteinflüsse und die sich andeutende Abhängigkeit von der genetischen Konstitution der Völker sollte diese Folgerung aber noch nicht verallgemeinert werden. Die Notwendigkeit einer Herbstbehandlung sollte stets im Einzelfall anhand der tatsächlichen Befallslage beurteilt werden. Dazu erscheint die befristete Erfassung (etwa 10-14 Tage) des natürlichen Milbentotenfalls im Verlauf des Spätsommers (Ende August, Anfang September) als praxisgerechte Möglichkeit. Wenngleich eine zuverlässige Hochrechnung im Sinne Liebigs et al. (1984) am Einzelvolk nach Ergebnissen von Rademacher (1985) nicht möglich ist, dürften die in der Folge mehrer Jahre für einen bestimmten Standort erhobenen Durchschnittswerte doch zu brauchbaren Anhaltspunkten führen.

Als besonderer Aspekt sei abschließend hervorgehoben, daß die vorgelegten Befunde die Möglichkeit eröffnen, im Falle einer sich eventuell abzeichnenden Resistenz gegen ein bestimmtes Mittel dieses gefahrlos über gewisse Zeit auszusetzen. Darüberhinaus bietet sich nunmehr die Möglichkeit, im Rahmen bestimmter Versuchsvorhaben Völker ohne jeglichen Mitteleinsatz über längere Zeit schadensfrei zu erhalten. 


\section{RÉSUMÉ \\ LA MÉTHODE DU RAYON PIĖGE COMME PARTIE INTÉGRANTE DE LA CONDUITE DU RUCHER EN CAS DE FORTE INFESTATION PAR VARROA JACOBSONI}

A lheure actuelle, en cas d'infestation par Varroa, l'apiculture ne peut se maintenir que par l'application régulière de traitements chimiques. Dans l'optique du développement éventuel de parasites résistants et la possibilité d'une augmentation constante de la contamination résiduelle du miel et de la cire, il est nécessaire de mettre au point des stratégies de lutte alternatives. Parmi les procédés biotechniques recommandés jusqu'à présent, la technique du rayon piège s'est révélée être la plus efficace. La reine est isolée pendant 3 périodes successives de 9 jours ou 4 de 7 jours sur un rayon d'ouvrières vide, renouvelé à chaque période (Fig. 1). On laisse chacun de ces rayons pièges dans la colonie durant une autre période pour qu'il soit operculé, puis on le retire avant lémergence et le détruit. Cet article présente des donnćes provenant d'expérimentations pratiques, qui semblent propres à améliorer l'adhésion de l'apicultcur, qui est resté jusqu'à présent très hésitant.

On a utilisé des colonies de carnoliennes ayant un faible taux d'infestation pour tester l'action de ce traitement sur le développement et la productivité ultérieurs. Les colonies expérimentales et les colonies témoins étaient placées dans les mêmes ruchers. Pour deux sćrics expérimentales, qui ont débuté en 1985 et 1986, on a mesuré les caractères suivants: la force de la colonie au début de l'expérience, avant l'hivernage et au début de la saison suivante ; la récolte de micl de la saison en cours et de la saison suivante. En 1986 on a également déterminé le temps de travail nécessaire.

Dans une expéricnce séparée, on a maintenu des colonies de carnoliennes ayant un taux élevé d'infestation avec la technique du rayon piège comme seule méthode de lutte contre Varroa. Le développement de la population de Varroa est donné par le taux de mortalité naturelle, enregistré tout au long de l'année grâce à des langes sur le plancher des ruches.

Les résultats sont les suivants :

1. Les colonies expérimentales ont tendance à être plus faibles avant l'hivernage de l'année de traitement et avant la saison de l'année suivante et à maintenir l'élcvage du couvain plus longtemps à l'automne que les témoins (Tabl. 1 et 2). Les différences ne sont pas significatives.

2. Dans le cas d'une bonne miclléc d’été, les colonies expérimentales ont tendance à produire légèrement plus de miel pendant la saison en cours que les témoins, mais légèrement moins la saison suivante (Tabl. 1 et 2). Là non plus, les différences ne sont pas significatives.

3. Le temps de travail néccssaire pour les deux premières étapes de la technique est environ deux fois plus important que pour des inspections normales (Fig. 2). On n'a pas pu mettre en évidence un avantage éventuel résultant de l'action anti-essaimage du traitement, en raison d'une tendance généralement faible à l'essaimage chez les témoins.

4. L'utilisation annuelle de la technique du rayon piège dans un rucher expérimental fortement infesté a semblé suffire comme seule mesure de lutte contre Varroa de mai 1985 à septembre 1987. Jusqu'à présent on n'a jamais pu observé de symptôme clinique. Néanmoins, à titre de précaution, six colonies fortement infestées ont été retirées. La récolte moyenne de miel par colonie a été de $47,7 \mathrm{~kg}$ en 1986 et de $29,5 \mathrm{~kg}$ en 1987 . Les chiffres du taux de mort naturelle des acariens indiquent qu'en moyenne leur population s'accroît d'année en année (Fig. 3a-c).

On a observé des différences significatives dans le taux d'infestation estimé entre des colonics d'origine génétique différente (Tabl. 3). Il n'est donc pas possible de faire des recommandations générales sur la durée pendant laquelle on peut suspendre sans danger les traitements chimiques. L'apiculteur doit être capable d'évaluer l'état d'infestation de ses ruchers pour prendre une décision. Pour ce faire, l'enregistrement du taux de mort naturelle des acariens à la fin de l'été devrait être le moyen le mieux approprić. 


\section{SUMMARY}

\section{THE TRAPPING-COMB TECHNIQUE AS PART OF BEE MANAGEMENT UNDER SEVERE INFESTATION BY VARROA JACOBSONI OUD.}

At present, beekeeping can be maintained during Varroa infestation only by mcans of regular application of chemical treatments. In view of the likely development of resistant parasites and the possibility of a steady increase in residuc contamination of honey and wax, alternative strategies of control must be developed. Among the biotechnical procedures recommended so far, the so-called trapping-comb-technique has turned out to be the most effective one. The queen is trapped for subsequent intervals of $3 \times 9$ days or $4 \times 7$ days on one empty worker comb per interval (Fig. 1). Each of these trapping combs remains in the colony for another interval to be scaled and is removed and destroyed before hatching. This paper presents data from practical testing. The results should improve acceptance of the technique by beekecpers, which has heretofore beer slight.

Carniolan colonics with a low infestation rate were used to test the influence of trapping-comb treatment on their further development and productivity. Experimental and control colonies were kept in the same apiarics. In two experimental series, starting in 1985 and 1986, the following characters were measured : the colony strength at the starting point, before wintering and at the beginning of the following season ; the honey yield from the current and following season. In 1986 the required working time was also determined.

In a separate experiment, begun in 1985, Carniolan colonies with a high infestation level were maintained with the trapping-comb-technique as the only measurc of Varroa control. The development of the Varroa population, as indicated by the natural death rate, was monitored throughout the year by means of screcned bottom sheets.

The results were as follows :

1. Experimental colonies tend to be weaker before wintering in the year of treatment and before next year's season and to maintain brood rearing longer into autumn than the controls (Table 1 and 2). The differences are not significant.

2. Under the conditions of a good summer honcy flow, experimental colonies tended to produce slightly more honey than the controls in 1985 but slightly less in 1986 (Table 1 and 2). Again, these differences are not significant.

3. The time required to complete the first two steps of the procedure is about two times higher than for regular inspections (Fig. 2). The effect of the treatment on swarming control was not established in the experiments because of generally low swarming tendency in the controls.

4. The annual application of the trapping-comb-technique appeared to be adequate as the only measure of Varroa control in a highly infested experimental apiary from May 1985 to September 1987. No clinical symptoms were observed. The average honey yicld per colony was $47.7 \mathrm{~kg}$ in 1986 and $29.5 \mathrm{~kg}$ in 1987. Six highly infested colonies were removed from the experiment in September 1987 because the infestation was too high for winter survival without chemical treatment. Measurement of the natural death rate of mites indicated a steady increase in mite populations from season to season (Fig. 3a-c).

Significant differences in the estimated rate of infestation were observed between colonies of different genetic origin (Tabl. 3). Therefore, no general recommendation is possible for the time span for which chemical treatments may be suspended without danger of losscs. The beekeeper must be able to evaluate the mite infestation levels within the apiary. For this purpose, measurement of the natural death rate of mites at intervals in late summer should be best suited. 


\section{LITERATUR}

Klepsch A., MaUl V., 1983. - Ncuc Versuche zur Wirksamkeit des Bannwabenverfahrens. Int. Bienenzucht Kongr. Apimondia, Bukarest, 258-265.

Kroos H., 1986. - Die Wildeshauser Betriebsweise. Selbstverlag (Haus am Kick, 2832 Rüssen).

LieBig G. et al., 1984. - Ergebnisse der Untersuchungen über dic Befallsentwicklung in StuttgartHohenheim 1983. Allg. disch. Imkerztg., 18, 185-190).

MaUL V., 1983 a. - Varroa-Elimination mittels Brutbeschränkung auf Bannwaben - Neuc Ergebnisse zur Wirksamkeit des Verfahrens. Apidologie, 14, 260-261.

MaUl V., $1983 \mathrm{~b}$. - Empfchlungen zur Methodik der Varroa-Elimination mittels Bannwaben aus Arbeiterbrut. Allg. dtsch. Imkerztg., 17, 179-194.

Pfefferie K.. 1983. - Verschiedene Verfahren zur Erzeugung varroafreier Nachwuchskolonien und dic laufende Erneuerung des Völkerbestandes durch das Rotationsprinzip. Allg. dtsch. Imkerztg., 17, $281-287$.

Preuß Ch., 1920. - Preuss'sche Imkerschule. Verlag T. Fischer, Leipzig, Berlin, Freiburg.

Rademacher E., 1985. - Ist eine Befallsprognose aus dem natürlichen Totenfall von Varroa jacobsoni möglich ? Apidologie, 16, 395-405.

RutTNFr F., Kofniger N., 1979. - Versuche zur Eliminierung der Varroa-Milben mit biologischen Methoden. Int. Bienenzucht Kongr. Apimondia Athen, 400)-402.

Ruttner F., Koeniger N., Ritter W., 1980. - Brutstop und Brutentnahme. Allg. disch. Imkerzig., 14, $159-160$.

Schulz A., Koeniger N., RuttNer F., 1983. - Drohnenbrut ats Varroafalle. Versuchsergebnisse 1981/ 1982. Allg. dtsch. Imkerztg., 17, 52-54.

Staemmi.:R G., 1986. - Das Bannwabenverfahren unter schleswig-holsteinischen Klimabedingungen. Die Neue Bienenzucht, 13, 11-14.

Staemmler G., 1987. - Das Bannwabenverfahren unter schleswig-holsteinischen Klimabedingungen. Die Neue Bienenzucht, 14, 94-98. 